\title{
Preparation and evaluation of high-lignin content cellulose nanofibrils from eucalyptus pulp
}

\author{
Martha Herrera $\cdot$ Kasinee Thitiwutthisakul Xuan Yang • Pim-on Rujitanaroj • \\ Ramiro Rojas $\mathbb{B} \cdot$ Lars Berglund
}

Received: 14 December 2017/ Accepted: 27 March 2018/Published online: 9 April 2018

(C) The Author(s) 2018

\begin{abstract}
High Klason lignin content (23 wt \%) cellulose nanofibrils (LCNF) were successfully isolated from eucalyptus pulp through catalyzed chemical oxidation, followed by high-pressure homogenization. LCNFs had a diameter of ca. $13 \mathrm{~nm}$ according to AFM evaluation. Dense films were obtained through vacuum filtration (nanopaper) and subjected to different drying methods. When drying under heat and mild vacuum $\left(93{ }^{\circ} \mathrm{C}, 95 \mathrm{kPa}\right)$ a higher water contact angle, lower roughness and oxygen transmission rate were observed, compared to those drying at room temperature under compression conditions. DSC experiments showed difference in signals associated to $\mathrm{T}_{\mathrm{g}}$ of LCNF compared to CNF produced from spruce bleached pulp through enzymatic pre-treatment. The LCNF-based nanopaper
\end{abstract}

Electronic supplementary material The online version of this article (https://doi.org/10.1007/s10570-018-1764-9) contains supplementary material, which is available to authorized users.

M. Herrera $\cdot$ X. Yang $\cdot$ R. Rojas $(\bowtie) \cdot$ L. Berglund Department of Fibre and Polymer Technology and Wallenberg Wood Science Center, KTH Royal Institute of Technology, 10044 Stockholm, Sweden e-mail: ramiro@kth.se

K. Thitiwutthisakul · P. Rujitanaroj ( $\square)$

Product and Technology Development Center, SCG

Packaging Public Company Limited, Ban Pong,

Ratchaburi 70110, Thailand

e-mail: pimonruj@scg.co.th showed mechanical properties slightly lower than for those made from cellulose nanofibrils, yet with increased hydrophobicity. In summary, the high-lignin content cellulose nanofibrils proved to be a suitable material for the production of low oxygen permeability nanopaper, with chemical composition close to native wood.

Keywords Lignocellulose nanofibrils · Oxygen barrier · Eucalyptus

\section{Introduction}

Wood is a versatile carbon-neutral material from renewable resources used through human history for many purposes ranging from combustion to construction to fine arts. Simply expressed, wood is an organic nanocomposite of cellulose nanofibrils embedded in a hydrated matrix of lignin and hemicellulose. Cellulose nanofibrils (CNF) are the reinforcement phase of the composite and have been isolated from different sources and studied as strengthening components in many material concepts (Abe et al. 2009; Abraham et al. 2011; Herzele et al. 2016; Sehaqui et al. 2010). CNF can be isolated from lignocellulosic biomass by enzymatic or chemical pretreatments followed by mechanical disintegration to form nanofibrils with lengths in the $0.5-3 \mu \mathrm{m}$ range and diameters from 3 to 
$30 \mathrm{~nm}$ (Henriksson et al. 2007; Ankerfors et al. 2013). A widely used chemical pretreatment for the isolation of CNF is TEMPO-mediated oxidation. With this pretreatment, carboxylate groups are selectively formed on the cellulose fibril surfaces without significantly altering their crystallinity. TEMPO-mediated oxidation has previously been reviewed in detail (Isogai et al. 2011). Even though CNF are widely studied as a reinforcement in composites (Pandey et al. 2013), cellulose by itself is hydrophilic, which makes it challenging to disperse in non-polar media and achieve strong interfacial interactions with commonly used polymers, e.g., polyethylene, polypropylene, or polystyrene due to poor compatibility (Herzele et al. 2016). The interface problem has been addressed through several different approaches (Abe et al. 2009; Habibi 2014), including the addition or preservation of lignin in cellulose fibrils due to its hydrophobicity (Abe et al. 2009). Compared to the linear chain of $\beta$ 1,4-linked D-glucose units in cellulose, lignin is a structurally complex macromolecule rich in phenylpropane units (Rinaldi et al. 2016).

Abe et al. (2009) demonstrated how chemi-thermomechanical pulp (CTMP) could be used to obtain a mixture of micro- and nano-sized fibers, after careful mechanical grinding and press-molding, to produce thermally plasticized composites without any additives or resins. Recently, Rojo et al. (2015), reported that $13.5 \mathrm{wt} \%$ of residual lignin in $\mathrm{CNF}$ nanopaper resulted in a reduction of their hydrophilicity, water vapor and gas permeability. Furthermore, Nair et al., demonstrated that lignin-containing cellulose nanofiber (LCNF) nanopaper had a reduced water uptake compared to their high-cellulose content CNF counterparts. Seemingly, the improved properties of these new materials are due to the hydrophobic lignin that fills space between the cellulose nanofibrils. Moreover, the lignin present in the nanopaper may decrease possibilities for water molecules to reach hydroxyl groups on cellulose fibrils, therefore reducing the extent of water-cellulose hydrogen bonds (Nair and Yan 2015).

The use of LCNF is an alternative to more traditional cellulose nanofibrils from bleached pulp and can potentially offer new uses and applications. Lignin can act as a compatibilizer to hydrophobic polymers and potentially improve their dispersion in a composite. Moreover, lignin and hemicellulose can act as natural glue when heated, filling porosities of nanopaper, reducing their permeability to gases and moisture. Furthermore, lignin has different types of chemical functionalities compared to cellulose, facilitating other modifications compared to those already existing.

In this study, LCNF were isolated from eucalyptus pulp under mild oxidation conditions followed by high-pressure microfluidization. The LCNF were used to produce nanopaper that were dried under different conditions. Chemical, morphological, thermal, barrier, and mechanical properties of the respective nanopaper were investigated. Delignified enzymatic $\mathrm{CNF}$ was used as the reference system. Therefore, the present study describes the effect of lignin and different drying conditions on the properties of nanopaper.

\section{Experimental}

Materials

Eucalyptus pulp from a semi-chemical pulping process-similar to chemo-thermo-mechanical pulping (SCG Packaging Public Company Limited, Thailand), was used for the isolation of the LCNF. The pulp had $26 \mathrm{wt} \%$ lignin, with a fiber length of $635 \mu \mathrm{m}$ and diameter of $15 \mu \mathrm{m}$. Enzymatically pre-treated CNF from never-dried sulphite pulp (enz-CNF) as described by Henriksson et al. (2007) was used as the reference system. Sodium hypochlorite (VWR International), sodium bromide (Sigma-Aldrich), 4-acetamido-TEMPO free radical, 4-AT (TCI Europe), sodium hydroxide pellets and hydrochloric acid (Fisher Scientific) were used as received.

\section{Pulp pre-treatments}

The eucalyptus pulp was diluted in distilled water to a concentration of $2 \mathrm{wt} \%$ and disintegrated in a laboratory pulp disintegrator (Noram, Canada) for 30,000 rotations. The disintegrated material was filtered and extracted with acetone and washed with hot deionized water under vacuum filtration conditions for four cycles. The extracted compounds are mainly lipophilic molecules which might have become more soluble after the semi-chemical pulping process. No fines were recovered during the filtration steps of the extraction process. The extracted pulp was then re-disintegrated 
and refined using a PFI mill (Norwegian Pulp and Paper Research Institute, Norway) for 2000 rotations.

\section{4-Acetamido-TEMPO (4-AT) mediated oxidation}

The 4-AT-mediated oxidation of extracted eucalyptus pulp was carried out as a starting point, by taking previously reported methods (Ma et al. 2010, 2012; Okita et al. 2010; Saito et al. 2010). The oxidation adds negative charges to the surface of the fibers which open up their structure and promote swelling and the possibility of isolating LCNF.

The pre-treated pulp was diluted to obtain $1.5 \mathrm{~L}$ of a $1 \mathrm{wt} \%$ suspension and overhead stirred at $150 \mathrm{RPM}$ while $6 \mathrm{mmol} / \mathrm{g}$ pulp of $\mathrm{NaBr}$ and $80 \mu \mathrm{mol} / \mathrm{g}$ pulp of 4-AT were added. The suspension was stirred for $5 \mathrm{~min}$ before $90 \mathrm{~mL}$ of $\mathrm{NaClO}$ (6 mmol/g pulp) was added drop-wise. The reaction time was $60 \mathrm{~min}$ from the moment when the $\mathrm{pH}$ reached 10.5. Thereafter, the $\mathrm{pH}$ was maintained at 10.5 by addition of $1 \mathrm{M} \mathrm{NaOH}$ and/or $0.5 \mathrm{M} \mathrm{HCl}$. After the reaction time, the reaction was quenched with ethanol and stirred under open system conditions for one more hour. The work-up was done at $\mathrm{pH} 2$ by filtering the oxidized fibers through a $15 \mu \mathrm{m}$ pore nylon mesh and washing until $\mathrm{pH}$ of filtrate was neutral.

Isolation of the high-lignin content cellulose nanofibrils

The oxidized pulp was diluted to $2 \mathrm{wt} \%$ and the $\mathrm{pH}$ raised to 8 . Thereafter, the solution was dispersed for $20 \mathrm{~min}$ at 12,500 RPM using a roto-stat ultra-turrax T25-D equipped with a S25N-18G dispersion element (IKA-Werke GmbH, Germany). The dispersed suspension was microfluidized using a Microfluidizer ${ }^{\circledR}$ Processor M-110 EH (Microfluidics, USA) for different cycles and pressures through different channel chamber systems: $1 \times 550 \mathrm{kPa}(1 \mathrm{~mm} \rightarrow 400 \mu \mathrm{m})$; $3 \times 5500 \mathrm{kPa} \quad(400 \mu \mathrm{m} \rightarrow 200 \mu \mathrm{m}) ; \quad$ and $3 \times 12,400 \mathrm{kPa}(200 \mu \mathrm{m} \rightarrow 100 \mu \mathrm{m})$. The obtained gel was fractionated by sedimentation. For this, a suspension of $0.2 \mathrm{wt} \%$ was left undisturbed at normal RT conditions for $72 \mathrm{~h}$. The upper phase was separated using a pipette and centrifuged at 4500 RPM for $20 \mathrm{~min}$ at $23{ }^{\circ} \mathrm{C}$. The supernatant was collected and centrifuged again at 9000 RPM for $20 \mathrm{~min}$ at $10{ }^{\circ} \mathrm{C}$. The supernatant obtained from this last centrifugation contained the final LCNF. The fractionation process and the yield for each step is summarized in Table 1.

Preparation of nanopaper

The LCNF nanopaper were made with $500 \mathrm{~mL}$ of a $0.05 \mathrm{wt} \%$ diluted LCNF suspension which was dispersed on an ultra-turrax for $10 \mathrm{~min}$ at $12,500 \mathrm{RPM}$. This suspension was then vacuum-filtered through a hydrophilic poly(vinylidene fluoride), PVDF, membrane with pore size of $0.65 \mu \mathrm{m}$ (Millipore, Germany) (Sehaqui et al. 2010). The obtained wet cakes were dried using different methods as described below.

Drying under temperature and vacuum (HT)

The wet cake obtained was covered with another PVDF membrane and dried using a semi-automatic sheet former Rapid Köthen (Blattbildner System PTI, Austria) at $93{ }^{\circ} \mathrm{C}$ and $95 \mathrm{kPa}$ vacuum for $15 \mathrm{~min}$.

Drying at normal room conditions under pressure (RT)

The wet cake obtained from the vacuum filtration was covered with another PVDF membrane and placed between 40 filter papers with a $4.5 \mathrm{~kg}$ plate on top for 5 days at room temperature conditions.

Freeze drying of samples (FD)

The wet cake obtained from the vacuum filtration was frozen by submerging in liquid nitrogen and then subjecting it to freeze drying conditions $\left(-83^{\circ} \mathrm{C}\right.$ and $0.262 \mathrm{hPa}$ vacuum) for $72 \mathrm{~h}$ to remove water by sublimation. Table 2 shows the description and names of the samples prepared and discussed through this study.

\section{Characterization methods}

Klason lignin and sugar analysis

The Klason lignin and sugar analysis were performed before and after the oxidation process. The Klason lignin content was measured according to the standard TAPPI T222 om-02, and the sugar content was measured after acid hydrolysis using a High- 
Table 1 Fractionation process and yields for the microfluidized pulp

\begin{tabular}{lll}
\hline Fractionation step & Process & Yield (\%) \\
\hline 0 & 4-AT mediated oxidation $\rightarrow$ microfluidized & 97 \\
1 & Decantation, sediment (bottom) & 19.1 \\
& Decantation, upper-phase (top) & 80.9 \\
2 & Centrifugation 1, pellet & 29.9 \\
& Centrifugation 1, supernatant & 70.1 \\
3 & Centrifugation 2, pellet & 14.9 \\
& Centrifugation 2, supernatant & 85.1 (48.5\% global yield) \\
\hline
\end{tabular}

Table 2 Summary of conditions for nanopaper based on the initial substrate and processing conditions

\begin{tabular}{lll}
\hline Fractionation step & Drying technique & Sample code \\
\hline 0 (unfractionated) & High temperature $\left(93{ }^{\circ} \mathrm{C}\right)$ and vacuum $(95 \mathrm{kPa})$ & Unfractionated-HT \\
& Room temperature under pressure & Unfractionated-RT \\
& Freeze dried & Unfractionated-FD \\
3 (colloidal stable) & High temperature $\left(93{ }^{\circ} \mathrm{C}\right)$ and vacuum $(95 \mathrm{kPa})$ & Fibrils-HT \\
& Room temperature under pressure & Fibrils-RT \\
& Freeze dried & Fibrils-FD \\
\hline
\end{tabular}

Performance Anion Exchange Chromatography (HPAEC) Dionex ICS-3000 by comparing to a reference of rhamnose, arabinose, galactose, glucose, xylose, and mannose.

Viscosity measurement of microfluidized suspensions

Microfluidized suspensions of unfractionated LCNF and enz-CNF with a concentration of $1.3 \mathrm{wt} \%$ were used for measuring their viscosity using a Brookfield viscometer DV2T extra (Middleboro, USA) and a spindle no. 73. The test was performed at $21{ }^{\circ} \mathrm{C}$ and 10 RPM for $5 \mathrm{~min}$. The reported results are the average of 3 different measurements.

Total charge determination

The total charge content of the LCNF was determined by polyelectrolyte titration (PET) using a Stabino Particle Charge Mapping (Microtrac Europe GbmH, Germany), with poly(diallyldimethyl ammonium chloride), PDADMAC, as cationic titrant (Uhlig et al. 2016). Additionally, the zeta potential, a measure of the colloidal stability of the solutions, and the estimated length of the fibrils, assuming the fibrils being spherical particles, were measured using a Zetasiser ZEN3600 (Malvern Instruments Ltd., UK).
Morphology of the fibrils and nanopaper

Fibrils suspensions were placed onto a plasma-treated silicon wafer and left to dry at room conditions. The fibril structure and LCNF nanopaper surface were observed using a field emission scanning electron microscopy (FE-SEM) Hitachi S-4800 (Japan). All samples were coated in a Cressington sputter coater 208HR with $5 \mathrm{~nm}$ thickness platinum: palladium (80:20) alloy. The morphology of the samples was also studied with an atomic force microscope Nanoscope V (Bruker Corporation, USA.) in tapping mode. The surface roughness $(\mathrm{Ra})$ was measured from the images obtained from analysis of nanopaper. The $\mathrm{Ra}$ reported is the average of the measurements done on 5 different images on a surface area of $1 \mu \mathrm{m}^{2}$. No image processing, except flattening, was used.

Static contact angle

The static contact angle for water was measured to study the hydrophilic/hydrophobic behavior of the LCNF. The contact angle was measured using a contact angle meter CAM20 (KSV Instruments, Finland). A $4 \mu \mathrm{L}$ drop of deionized water was placed onto the surface of the nanopaper and the contact angle was calculated after $10 \mathrm{~s}$. The reported contact angles are the average of 3 measurements. 
Thermal properties of nanopaper

The thermogravimetrical analysis (TGA) of the nanopaper was studied using a TGA/DSC1 $\mathrm{STAR}^{\mathrm{e}}$ System from 120 to $800{ }^{\circ} \mathrm{C}$ at a rate of $10{ }^{\circ} \mathrm{C} / \mathrm{min}$. Differential scanning calorimetry (DSC) measurements were performed using a DSC1 STAR ${ }^{\mathrm{e}}$ system (Mettler Toledo, Switzerland). Samples weighing around $3 \mathrm{mg}$ from room temperature to $275^{\circ} \mathrm{C}$ at a rate of $10{ }^{\circ} \mathrm{C} / \mathrm{min}$. For this test, the samples used were freeze-dried, in order to investigate the thermal properties of the materials at different conditions. All tests were done under inert nitrogen atmosphere.

Fourier transformed infrared studies

FT-IR spectroscopy was performed on a Perkin-Elmer Spectrum 2000 FT-IR equipped with a MKII Golden Gate, Single Reflection ATR system (Specac, UK) at room temperature. Eight scans were carried out from 4000 to $600 \mathrm{~cm}^{-1}$ wavenumbers.

Oxygen barrier properties

The oxygen transmission rate (OTR) of the nanopaper at 30,50 and $90 \%$ relative humidity $(\mathrm{RH})$ were measured using an OX-TRAN ${ }^{\circledR}$ Model 2/20 (Mocon, USA) following the ASTM-3985 standard. The samples were conditioned at $23{ }^{\circ} \mathrm{C}$ and $50 \% \mathrm{RH}$, under ambient atmospheric pressure.

Mechanical testing

The LCNF nanopaper was tested in tension mode on a universal tensile testing system (Instron 5944, UK) equipped with a $500 \mathrm{~N}$ load cell. The specimens tested were stripe of $5 \mathrm{~mm}$ width, and a gauge length of $20 \mathrm{~mm}$ was used with a cross-head displacement of $10 \% / \mathrm{min}$. The tests were made in a conditioned room $\left(23{ }^{\circ} \mathrm{C}\right.$ and $50 \% \mathrm{RH}$ ), and the samples were conditioned for at least $48 \mathrm{~h}$ before the test. Between 5 and 7 specimens were tested for each nanopaper, and the reported data represents the average of these values.

\section{Results and discussions}

Extraction and mechanical pre-treatment

The eucalyptus pulp received from a semi-chemical pulping process was extracted with acetone. An average loss of around $15 \mathrm{wt} \%$ was observed corresponding to fines and extractives. The pulp fibers were initially $15 \mu \mathrm{m}$ in diameter and $635 \mu \mathrm{m}$ in length, and after extraction, disintegration and refining, fibrillation on the surface of the fibers was observed (more information available in the supplementary information).

The pre-treatment is used to facilitate the disintegration of the fibrils and decrease the energy demand of the isolation process (Jonoobi et al. 2015; Nechyporchuk et al. 2016). The extraction and mechanical pre-treatment are beneficial towards the chemical treatment. This facilitates the diffusion of the reagent through the pulp fibers and improves the surface modification of the fibers. Fines may decrease the degree of oxidation through the consumption of chemicals.

Klason lignin and sugar analysis

The Klason lignin and sugars composition before and after the 4-AT-mediated oxidation was measured and is summarized in Table 3.

After the 4-AT-mediated oxidation, the relative amount of glucose and xylose increased by about $5 \%$, while the relative amount of arabinose, galactose, mannose and rhamnose decreased, presumably due to hydrolysis of pectin and hemicellulose at alkaline conditions. The amount of Klason lignin decreased by about 5\%, however, the absolute amount of lignin (23 $\mathrm{wt} \%$ ) is much higher than other studies (Rojo et al. 2015; Nair and Yan 2015).

Microfluidization effect on fibril morphology

The effect on fibril morphology during each step of the microfluidization treatment was studied. Figure 1a shows the 4-AT mediated oxidized fibers prior to fibrillation. The fibers are long and with diameters of around $10-15 \mu \mathrm{m}$. They also seem porous and with irregular diameters along the length of the fiber. Figure $1 \mathrm{~b}$ shows the oxidized pulp bursting into fiber fragments and fibril aggregates after microfluidization 
Table 3 Chemical composition of eucalyptus pulp fibers

\begin{tabular}{llllllll}
\hline Pulp sample & Arabinose $(\%)$ & Galactose $(\%)$ & Glucose $(\%)$ & Mannose $(\%)$ & Xylose $(\%)$ & Rhamnose $(\%)$ & Klason lignin $(\%)$ \\
\hline As received & 0.35 & 1.50 & 59.78 & 0.54 & 6.98 & 0.10 & 28.45 \\
Oxidized & 0.12 & 0.96 & 63.82 & 0.35 & 11.73 & 0.05 & 22.95 \\
\hline
\end{tabular}
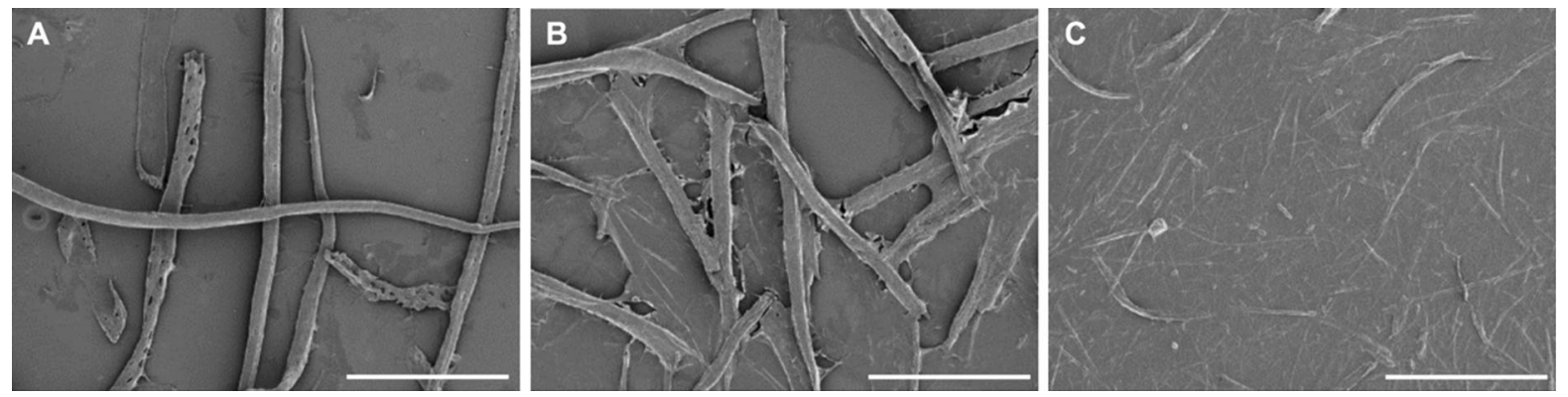

Fig. 1 SEM images of the eucalyptus pulp after 4-AT-mediated oxidation (a), after first microfluidization at $5500 \mathrm{kPa}$ (b), and after last step at $12,400 \mathrm{kPa}(\mathbf{c})$; scale bar is $100 \mu \mathrm{m}$

at $5500 \mathrm{kPa}$. After this step, the fibers are shortened but with similar diameters as in Fig. 1a. By the final microfluidization step (Fig. 1c) the obtained material is composed of shorter fibers with lengths of around $100 \mu \mathrm{m}$ and diameters in the micrometric and nanometric range. In order to isolate the nanofibrils from the microfibrils, further fractionation was carried out.

Qualitatively, it was noticed the microfluidization process produced viscous suspensions (supplementary information, Fig. S2). However, the measured viscosity of LCNF suspensions was $12,323 \pm 219 \mathrm{cP}$ while for enz-CNF 27,033 $\pm 803 \mathrm{cP}$. It is believed that the presence of lignin and hemicelluloses in eucalyptus oxidized pulps prevented higher defibrillation, swelling and dispersion compared to enz-CNF that does not contain lignin.

Fractionation, dimensions and colloidal stability of the LCNF

As seen in Fig. 1c, the final microfluidized suspension was a mixture of microfibrillated and nanofibrillated LCNF. In order to isolate the nanofibrils, the fractionation process described in Table 1 was performed. After fractionation, the fibrils length decreased from several micrometers to under micron-sized lengths based on dynamic light scattering approximations. Moreover, the zeta potential of the suspensions was
-45 and $-60 \mathrm{mV}$ before and after fractionation respectively. The zeta potential indicates the degree of electrostatic repulsion between particles in a dispersion, which is a measure of the colloidal stability. In general, if a colloidal suspension has a zeta potential $\geq 30 \mathrm{mV}$ or $\leq-30 \mathrm{mV}$, the suspension is stable (Isogai et al. 2011). The fact that the zeta potential values are lower than $-30 \mathrm{mV}$, indicates that the LCNF will remain suspended at equilibrium in the aqueous phase.

\section{Total charge determination}

In order to determine the charge of the LCNF fibrils after the fractionation, polyelectrolyte titration was performed. The LCNF fibrils had a charge of $857 \mu \mathrm{eq} /$ g pulp. An oxidation reaction with similar conditions on pure cellulose pulps, e.g., dissolving pulp or bleached Kraft pulp would yield a charge in the range between $800-1200 \mu \mathrm{eq} / \mathrm{g}$ pulp (Okita et al. 2009). It is believed that the lower carboxylate contents in the pulp and the reduction of the lignin content after the oxidation, see Table 3 , is due to $\mathrm{NaClO}$ reacting with the lignin, in a bleaching type of process, rather than to form carboxylates on the surface. This is supported by the change of color of the pulp from dark brown to a lighter orange shade. Moreover, as some oxidation is promoted on hemicellulose, its solubilization 
increases. Some hemicellulose may be dissolved, allowing some lignin to be removed in the form of lignocellulose complexes. These results suggest that the removal of fines during the extraction pretreatment is favorable in order to avoid overconsumption of chemicals. It may also reduce cellulose depolymerization from limited stability of glycoside bonds at high $\mathrm{pH}$ and with the presence of $\mathrm{NaClO}$ (Watanabe et al. 2013).

\section{Morphology of LCNF and nanopaper}

The supernatant obtained after the fractionation process, was investigated by AFM in order to estimate the diameter of the nanofibrils. Representative AFM images are presented in Fig. 2, where fine fibrils in the range of $3 \mathrm{~nm}$ in diameter are observed as well as other larger particles, that are believed to be fibril aggregates or non-fibrillated fiber bundles. These fiber bundles are in the range of $27 \mathrm{~nm}$ in diameter. The average diameter size was $13 \pm 8 \mathrm{~nm}$, thereby corroborating the nanoscale of the fibrils obtained. Carrillo et al. obtained similar size fibril bundles when preparing LCNF from Kraft digestion of eucalyptus using aqueous solutions of urea (Carrillo et al. 2014).

The nanopaper produced were observed by AFM and FE-SEM in order to measure roughness and observe surface structure. Representative AFM height images of the nanopaper are shown in Fig. 3.

Globular-shaped patches are apparent in Fig. 3a-c, some of them enclosed in circles. These patches are likely to be lignin agglomerates. Similar structures were seen and reported previously by different authors
(Rojo et al. 2015; Nair and Yan 2015; Börås and Gatenholm 1999). Nanopaper Unfractionated-HT (Fig. 3a) show thick particles and tight packing with a roughness of $31 \pm 7 \mathrm{~nm}$ in an area of $1 \mu \mathrm{m}^{2}$. It is important to notice that, for the rms roughness measurements, the areas with globular patches were avoided as much as possible. Nanopaper Unfractionated-RT were not prepared due to the large size of fibers and lignin agglomerates, resulting in more porous and rougher nanopaper. In nanopaper FibrilsHT (Fig. 3b), a network composed of fine fibers with homogeneous rms roughness of $16 \pm 4 \mathrm{~nm}$ is observed. However, much larger patches can be seen in the AFM images of nanopaper Fibrils-RT (Fig. 3c). These larger lignin particles increased the rms roughness and standard deviation to $30 \pm 16 \mathrm{~nm}$. The fact that the lignin particles appear to be frequent and of large size when the nanopaper is dried at room temperature, indicates that the HT drying process allows for a plasticization/flow of the lignin and hemicellulose in the nanopaper, which results in smoother surfaces upon drying. A more uniform structure with smaller globules are present in the samples with finer fibrils and dried at high temperature (Fig. 3b nanopaper Fibrils-HT). It is interesting to note that the lignin patches seem to be attached to the surrounding matrix, and not segregated as in previous reports (Rojo et al. 2015; Nair and Yan 2015; Börås and Gatenholm 1999). This possibly means that the lignin is associated with the cellulose fibrils, but more evidence is needed to support this observation regarding the isolated nanofibrils, and is out of the scope of the present article.
Fig. 2 AFM height images of $10 \times 10 \mu \mathrm{m}^{2}$ (left) and $3 \times 3 \mu \mathrm{m}^{2}$ (inset) of LCNF after fractionation
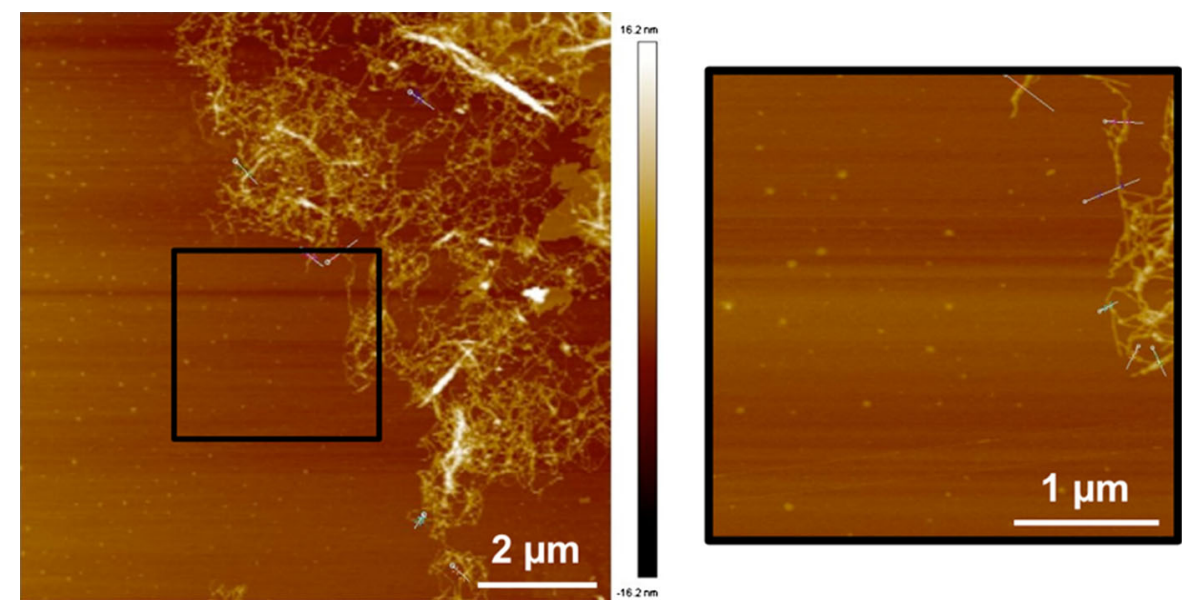

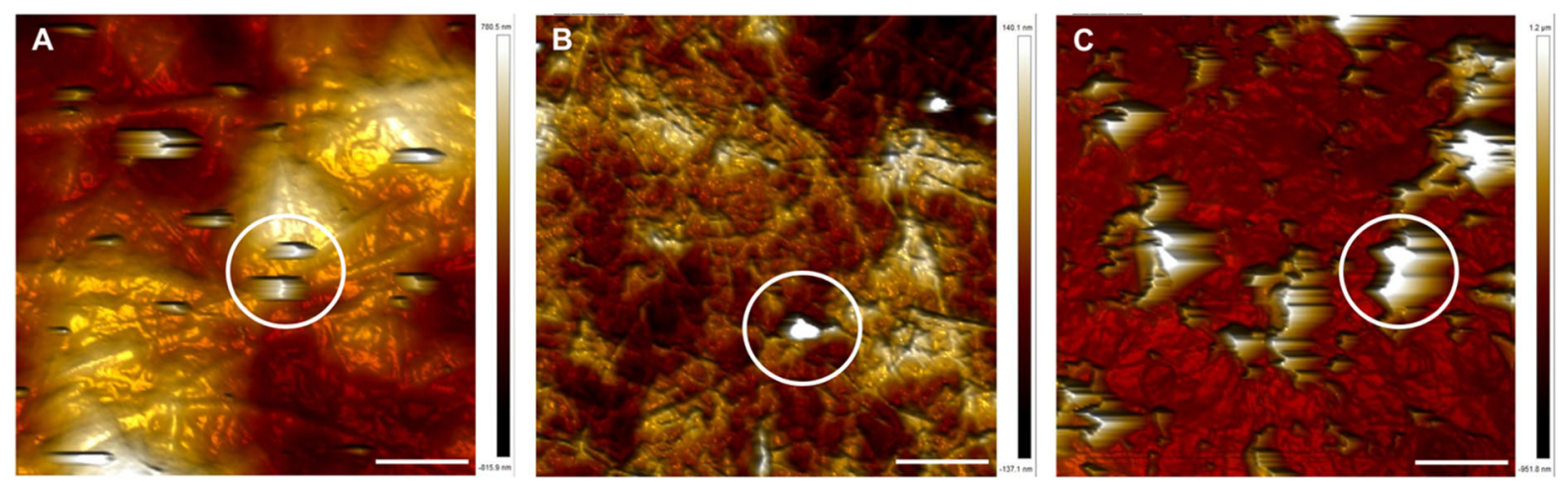

Fig. 3 AFM images of nanopaper Unfractionated-HT (a), Fibrils-HT (b), and Fibrils-RT (c), scale bar is $2 \mu \mathrm{m}$

The SEM images of nanopaper Fibrils-HT and nanopaper Fibrils-RT can be seen in Fig. 4. Figure 4a, c had similar magnification as the AFM images in Fig. 3 and similar patches can also be observed. In Fig. 4d a fibril network is more evident, with randomly oriented fibrils and some thicker fibril bundles. In Fig. 4b the fibrils are not as apparent and the structure seems to be more uniform and less porous, confirming that the processing conditions help to promote some type of flow of the lignin and the hemicellulose.
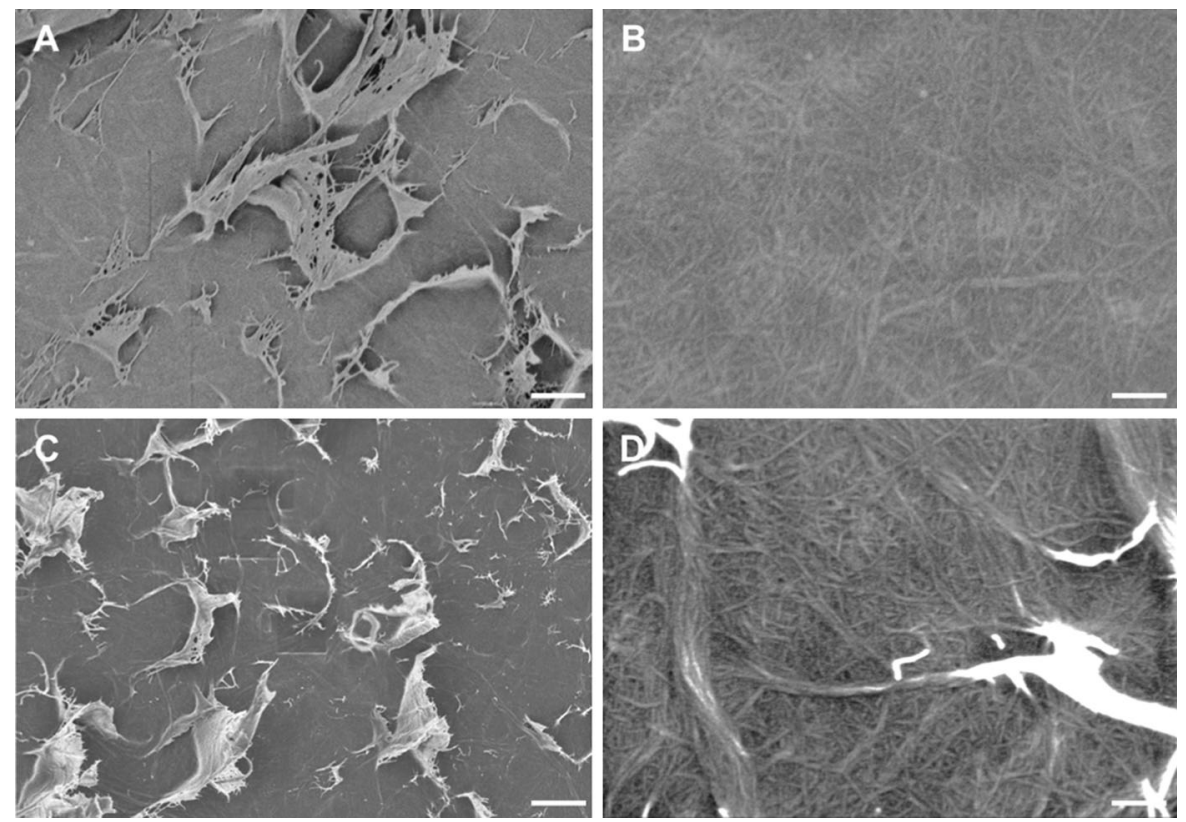

Fig. 4 FE-SEM images of nanopaper Fibrils-HT (a, c) and Fibrils-RT (b, d) at $10 \mathrm{~K}$ magnification and $100 \mathrm{~K}$; scale bars are $1 \mu \mathrm{m}$ and $100 \mathrm{~nm}$ respectively 
nanopaper Fibrils-HT was the highest and in the hydrophobic range. We suspect that the main reason for this is the difference in the morphology of the samples, particularly surface roughness as discussed above. Nanopaper based on fractionated fibrils tend to have lower surface roughness. Another reason for nanopaper fibril-HT to have higher contact angle is the more hydrophobic surface, caused by the flow of the lignin and hemicellulose, which might distribute more evenly across the surface and. Similar flow and plasticization processes were observed by Abe et al. in the range of $120-180{ }^{\circ} \mathrm{C}$ (Abe et al. 2009). In our case, it seems that the temperature and humidity used in the drying process decreased the $\mathrm{T}_{\mathrm{g}}$ of the lignin and made flow possible.

\section{Thermal properties of the LCNF nanopaper}

The thermal stability of Fibrils-HT and Fibrils-RT nanopaper was studied and compared to enz-CNF dried under the same HT conditions, as described above. The $\mathrm{T}_{\text {onset }}$ is defined as the temperature at which the sample weight loss becomes more apparent, and $T_{\max }$ is the maximum value of the $\mathrm{dm} / \mathrm{dT}$ derivative weight curve and represents the temperature at which the decomposition is the fastest (Nair and Yan 2015).

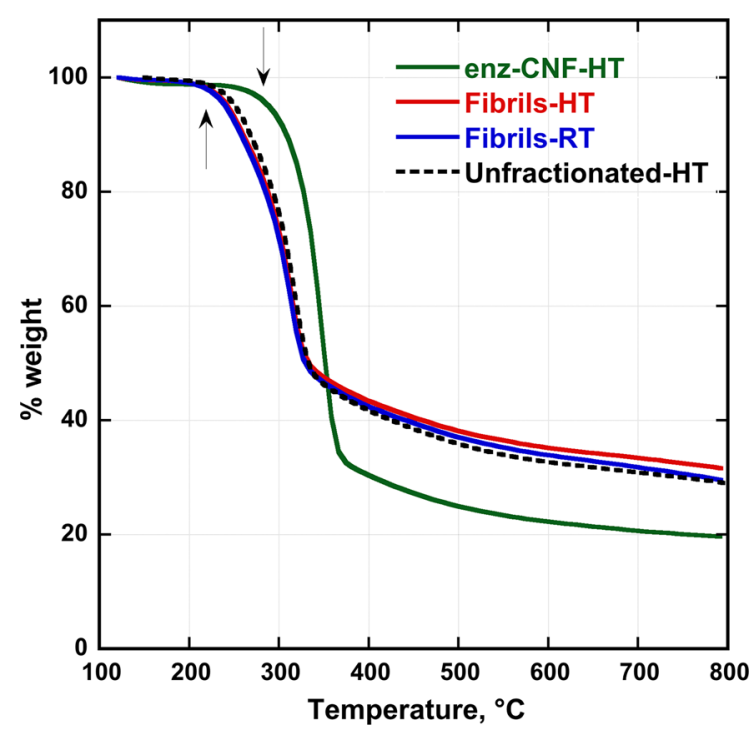

Fig. 5 TGA curves for Unfractionated-HT, Fibrils-HT, Fibrils$\mathrm{RT}$, and enz-CNF-HT nanopaper, the arrow point at the $\mathrm{T}_{\text {onset }}$ discussed in the main text
Figure 5 shows the thermal decomposition curves. Samples Unfractionated-HT, Fibrils-HT, Fibrils-RT presented very similar curves with a $\mathrm{T}_{\text {onset }}$ around $230{ }^{\circ} \mathrm{C}$, and a $\mathrm{T}_{\max }$ of about $300{ }^{\circ} \mathrm{C}$ and residue at $800{ }^{\circ} \mathrm{C}$ of around $30 \%$ and were less thermally stable than the reference. Lignin is known to have a low reactivity in oxygen deficient atmospheres at high temperature (Vänskä et al. 2016), which should provide protection to the LCNF nanopaper and increase thermal stability. However, the opposite behavior was observed, and the lignin rich nanopaper had lower thermal stability than the lignin-free enzCNF-HT nanopaper. This may to be due to the carboxylate groups introduced during the 4-ATmediated oxidation process. Although the carboxylate groups increase the colloidal stability of the LCNF suspensions, they also promote thermal degradation at lower temperatures (Fukuzumi et al. 2009, 2010). When comparing the $\mathrm{T}_{\text {onset }}$ and $\mathrm{T}_{\max }$ of LCNF with the values obtained for TEMPO-oxidized cellulose $\left(\mathrm{T}_{\text {on- }}\right.$ set $=222{ }^{\circ} \mathrm{C}$ ) by Fukuzumi et al (2010), it can be seen that LCNF has a higher thermal stability.

Studies on the pyrolysis of cellulose, hemicellulose and lignin have shown that the decomposition of cellulose starts at around $300{ }^{\circ} \mathrm{C}$ and reaches its maximum weight loss rate at around $350{ }^{\circ} \mathrm{C}$, similar to the present values for enz-CNF-HT with a $\mathrm{T}_{\text {onset- }}$ $=290{ }^{\circ} \mathrm{C}$. For hemicellulose and lignin, these temperatures decrease, starting the decomposition of hemicellulose at $220{ }^{\circ} \mathrm{C}$ and lignin below $200{ }^{\circ} \mathrm{C}$, and a maximum mass loss rate at $270{ }^{\circ} \mathrm{C}$ for hemicellulose while for lignin a continued weight loss up to $700{ }^{\circ} \mathrm{C}$ has been reported (Yang et al. 2007). The differences in the chemical compositions between the LCNF and enz-CNF nanopaper, produce the difference in their thermal properties. Since the LCNF has $23 \%$ lignin, a reduction in the thermal stability of the whole system is observed. However, the solid residue after the pyrolysis of Fibrils-HT and FibrilsRT were 50\% higher than for enz-CNF-HT, due to the lignin. Moreover, similarities in the curve profile in TGA and the residue at $800{ }^{\circ} \mathrm{C}$ indirectly support a similar composition for unfractionated and fractionated samples. Lignin has a reported solid residue (char) of $46 \%$ at $900{ }^{\circ} \mathrm{C}$ (Bourbigot and Le Bras 2004).

Differential scanning calorimetry (DSC) was performed to compare the thermal behavior of LCNF and enz-CNF in order to visualize any transitions at the 
nanopaper drying temperatures used in this study. The traces for dry samples can be seen in Fig. 6. At these conditions, inflection points marked by arrows at 52, 110, and $170{ }^{\circ} \mathrm{C}$ can be observed for Fibrils-FD, but only at $110{ }^{\circ} \mathrm{C}$ for enz-CNF-FD. These signals may correspond to thermal phenomena associated to glass transition, but they could represent other phenomena due to low heat flow and the complexity of the materials. The $\mathrm{T}_{\mathrm{g}}$ of lignin is reported to be about $135{ }^{\circ} \mathrm{C}$ in dry conditions (Abe et al. 2009; Bouajila et al. 2006) and, in the presence of water, it can be reduced to $80-90{ }^{\circ} \mathrm{C}$, due to the plasticization (Rojo et al. 2015). A similar reduction in the $T_{g}$ of cellulose has been seen in the presence of water (Szczesniak et al. 2008). This plasticization/flow effect has been previously studied in the processing range of 120-180 ${ }^{\circ} \mathrm{C}$, and it was concluded that lignin could be thermally plasticized without the use of any resins or adhesives (Abe et al. 2009). However, in the presence of moisture (data not shown) we were not able to identify any strong signals associated to $T_{g}$ as these signals could be out of the studied temperature range. The fact that no exothermic peaks were seen at dry or under $50 \%$ RH suggests that no chemical reactions (esterification, phenyl-phenyl crosslinks) are taking place prior to thermal degradation.

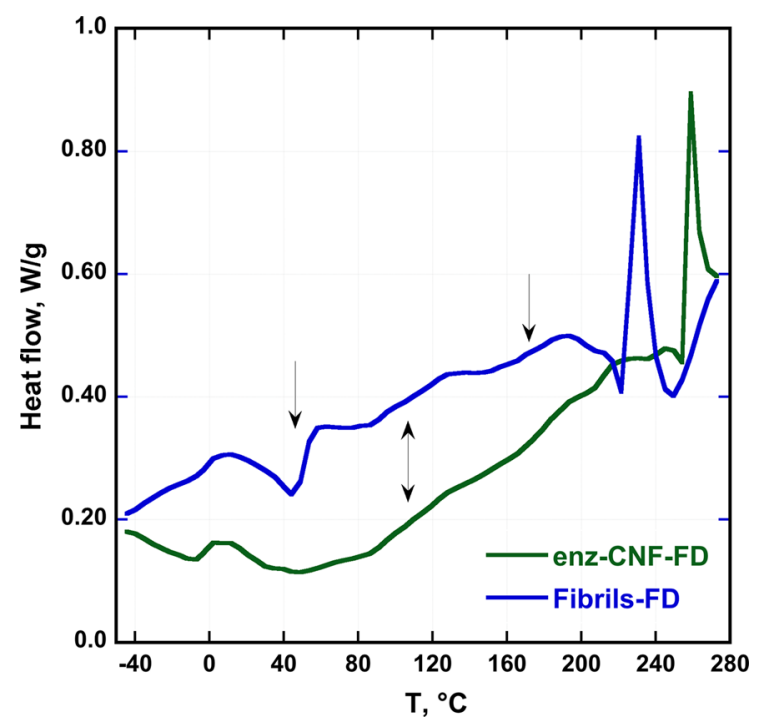

Fig. 6 DSC traces for Fibrils-FD, and enz-CNF-FD at dry conditions. Arrows mark inflection points discussed in the main text
Fourier transformed infrared spectroscopy

Figure 7 shows the FT-IR spectra of UnfractionatedFD, Fibrils-FD and enz-CNF-FD normalized in the range of $1200-900 \mathrm{~cm}^{-1}$. We only chose samples prepared using freeze-drying here, since RT and HT may disturb the thermal properties of the fibrils components. For LCNF, all typical infrared peaks for cellulose, hemicellulose and, lignin can be observed compared to enz-CNF-FD, particularly the lignin signature for aromatic skeletal mode at $1505 \mathrm{~cm}^{-1}$ (I on graph) (Yang et al. 2007; Szczesniak et al. 2008). The adsorption peak associated to the $\mathrm{O}_{-}$ $\mathrm{H}$ stretching vibration (around $3340 \mathrm{~cm}^{-1}$ ) is lower for the LCNF Unfractionated-FD compared to FibrilsFD which can be traced back to the oxidation reaction, where the larger fibers were mostly affected. When these larger fibers were removed in the fractionation process, the relative amount of $-\mathrm{COONa}$ groups was similarly removed. At $1600 \mathrm{~cm}^{-1}$ (II on graph) the adsorption peak associated with $\mathrm{C}=\mathrm{O}$ stretching vibration of the carboxylate groups can be observed. This peak is not evident in the reference material as the carboxylate groups result from the oxidation reaction. The peak observed at $1730 \mathrm{~cm}^{-1}$ (III on graph) is associated to residual free carboxyl groups (Fukuzumi et al. 2010) and it is smaller for nanopaper Fibrils-FD and in the reference, indicating the presence of very little amount of carboxyl groups in these samples. This peak is also associated to hemicellulose, indicating a lower amount in nanopaper enz-CNF-FD. From the

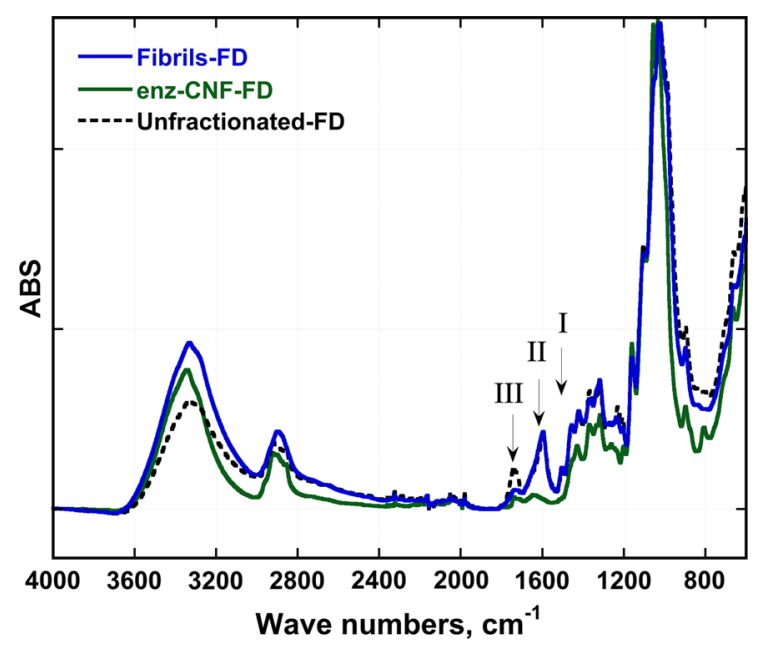

Fig. 7 FT-IR spectra for nanopaper Unfractionated-FD, Fibrils-FD and enz-CNF-FD 
FT-IR of the freeze dried nanopaper it can be concluded that nanopaper Unfractionated-FD and Fibrils-FD have similar chemical composition, and that both retained the native lignin present in the eucalyptus pulp.

\section{Oxygen transmission rate (OTR)}

An interesting experiment to perform for LCNF is the oxygen transmission rate of nanopaper due to its fibrillar morphology and partially thermoplastic properties. Initially, the OTR was tested in nanopaper Fibrils-RT and -HT at 50\% RH in order to compare the effect of processing and drying. It is noted that the OTR at $50 \%$ RH of Fibrils-RT was twice as high as the value obtained for Fibrils-HT $(0.19$ vs. 0.42 $\left.\mathrm{cc} \times \mathrm{mm} \times \mathrm{m}^{-2} \times \mathrm{day}^{-1} \times \mathrm{atm}^{-1}\right)$. This result supports the flow/plasticization of the lignin under HT drying conditions, increasing the oxygen barrier properties of the nanopaper. Lignin is known to plasticize at a range of temperatures between 120 and $180{ }^{\circ} \mathrm{C}$ (Abe et al. 2009), however, in the presence of water, this temperature can be reduced to $80-90{ }^{\circ} \mathrm{C}$ (Rojo et al. 2015), as seen in our LCNF nanopaper. Furthermore, the OTR values were tested for unfractionated samples and compared to the colloidal stable fibrils at different \% RH. Figure 8 shows the OTR values obtained for the nanopaper

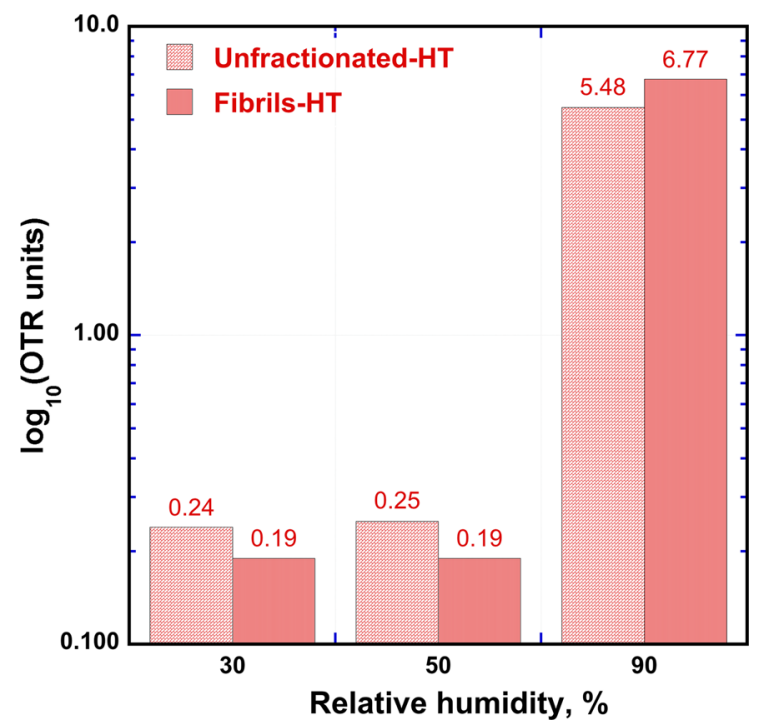

Fig. 8 OTR at 30, 50 and $90 \%$ RH for nanopaper Unfractionated-HT and Fibrils-HT OTR units $=\mathrm{cc} \times \mathrm{mm} \times \mathrm{m}^{-2} \times$ day $^{-1} \times$ atm $^{-1}$
Unfractionated-HT compared to Fibrils-HT at 30, 50 and $90 \% \mathrm{RH}$.

At 30 and $50 \%$ RH the OTR values are very similar between the nanopaper Unfractionated-HT, FibrilsHT, but at higher humidity is increased from about 0.2 to 6.7 OTR units. Surprisingly, the highest OTR value obtained (6.77 OTR units for Fibrils-HT at 90\%RH), is in the range for commercially available polymers such as poly(ethylene terephthalate), PET (1-5 OTR units), or polystyrene (99-150 OTR units) at 50\% RH (Lange and Wyser 2003). The suitability of nanocellulose as a gas barrier material is attributed to its ability to create dense structures (Herrera et al. 2016) of high crystalline order as the ones seen in Fig. 4. At high humidity, the amorphous part of the cellulose fibril absorbs water, increasing the polymer chain mobility and creating free volume for the oxygen permeation (Aulin et al. 2010). This process increases the OTR of the cellulosic materials at high RH similarly seen for the samples at $90 \% \mathrm{RH}$.

Tensile testing of the nanopaper

The tensile properties of the nanopaper Unfractionated-HT, Fibrils-HT and Fibrils-RT were measured at $23{ }^{\circ} \mathrm{C}$ and $50 \% \mathrm{RH}$ and the results for representative curves of stress versus strain can be seen in Fig. 9, a summary of the average Young's modulus, ultimate

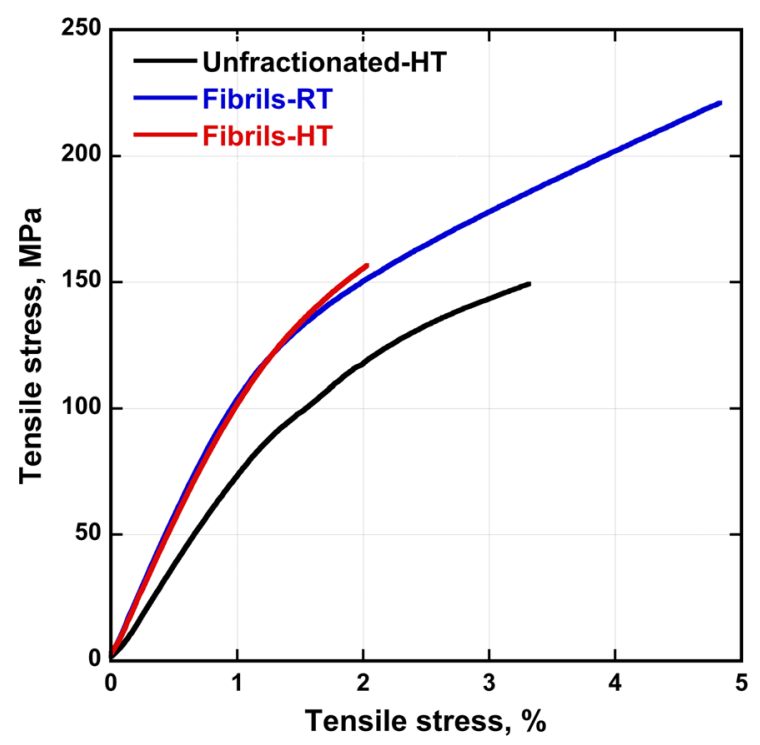

Fig. 9 Representative stress-strain curves for LCNF nanopaper Unfractionated-HT, Fibrils-HT and Fibrils-RT 
Table 4 Summary of the mechanical properties of LCNF nanopaper as obtained from uniaxial tensile tests

\begin{tabular}{lcll}
\hline Sample & Young's modulus $(\mathrm{GPa})$ & Ultimate strength $(\mathrm{MPa})$ & Strain to failure $(\%)$ \\
\hline Unfractionated-HT & $7.61 \pm 0.31$ & $143.68 \pm 5.99$ & $3.35 \pm 0.43$ \\
Fibrils-RT & $10.30 \pm 0.50$ & $184.21 \pm 50.20$ & $3.87 \pm 1.78$ \\
Fibrils-HT & $11.30 \pm 0.55$ & $137.78 \pm 35.40$ & $1.70 \pm 0.80$ \\
\hline
\end{tabular}

strength and strain at failure are presented on Table 4. The highest value for the Young's modulus was obtained for nanopaper Fibrils-HT with $11.1 \mathrm{GPa}$, and the lowest was for nanopaper Unfractionated-HT with 7.61 GPa. The strain at failure was around $3 \%$ for all samples except for nanopaper Fibrils-HT, which had an elongation closer to $2 \%$. The stress at break for nanopaper Unfractionated-HT and Fibrils-HT was very similar at around $140 \mathrm{MPa}$, however this value increased by almost $30 \%$ for nanopaper Fibrils-RT (184 MPa). This result suggests that when the nanopaper was dried at HT, their properties were lowered in comparison to the nanopaper dried at RT. The stress at break for the LCNF nanopaper was lower than for enzymatic or TEMPO-CNF without lignin (around $250 \mathrm{MPa}$ ) (Isogai et al. 2011). This is most likely due to the presence of the larger particles seen in the AFM (Fig. 3) and SEM (Fig. 4) images. They may act as stress concentrators, decreasing the overall stress at break of the LCNF nanopaper. Overall, LCNF can be considered a nanocomposite by itself and at a cellulose nanofibrils content of around $60 \%$, still shows good mechanical properties compared to higher cellulose content nanomaterials.

\section{Conclusions}

In this work, the isolation of lignin-rich cellulose nanofibrils has been developed using an oxidation pretreatment procedure often used in lignin-free cellulose pulp systems. Properties were analyzed before and after fractionation and after different drying processes to prepare nanopaper. 4-AT-mediated oxidation proved to be an effective pretreatment for disintegration of lignin-rich cellulose nanofibrils (LCNF) from eucalyptus pulp. The present lignin content (23 wt $\%$ ) was much higher than any previously published studies. The lignin in the systems acts as glue material between the cellulose fibrils in the nanopaper, just as it does in native wood. This is particularly true after drying the nanopaper at $93{ }^{\circ} \mathrm{C}$, where smoother surfaces presumably due to a flow/plasticization process were observed. When these nanopaper structures were dried at high temperature, they formed hydrophobic surfaces with oxygen permeability values even lower than for some commercially used polymers such as PET or polystyrene. The mechanical properties of the LCNF nanopaper dried at different conditions were comparable with those from fully bleached CNF although slightly lower. LCNF is a native wood composite nanofibril, which is available as an alternative to more pure cellulose nanofibrils. Moreover, the increased hydrophobicity makes these lignin-rich cellulose nanofibrils an appealing constituent for large-scale use in polymer biocomposites.

Acknowledgments $\mathrm{MH}$ would like to thank SCG Packaging Public Company Limited for supplying the raw materials and for the financial support through a post-doctoral fellowship. The Knut and Alice Wallenberg foundation through the Wallenberg Wood Science Center at KTH Royal Institute of Technology (XY, RR, LB) is also acknowledged for their continuous support.

Open Access This article is distributed under the terms of the Creative Commons Attribution 4.0 International License (http:// creativecommons.org/licenses/by/4.0/), which permits unrestricted use, distribution, and reproduction in any medium, provided you give appropriate credit to the original author(s) and the source, provide a link to the Creative Commons license, and indicate if changes were made.

\section{References}

Abe K, Nakatsubo F, Yano H (2009) High-strength nanocomposite based on fibrillated chemi-thermomechanical pulp. Compos Sci Technol 69:2434

Abraham E, Deepa B, Pothan L, Jacob M, Thomas S, Cvelbar U, Anandjwala R (2011) Extraction of nanocellulose fibrils from lignocellulosic fibres: a novel approach. Carbohydr Polym 86:1468 
Ankerfors M, Lindström T, Henriksson G (2013) US 20090221812 A1

Aulin C, Gällsted M, Lindström T (2010) Oxygen and oil barrier properties of microfibrillated cellulose films and coatings. Cellulose 17:559

Börås L, Gatenholm P (1999) Surface composition and morphology of CTMP fibers. Holzforschung 53:188

Bouajila J, Dole P, Joly C, Limare A (2006) Some laws of a lignin plasticization. J Appl Polym Sci 102:1445

Bourbigot S, Le Bras M (2004) Chapter 5. In: Troitzsch J (ed) Plastics flammability handbook principles, regulations, testing, and approval, 3rd edn. Hanser Publisher, Munich, p 133

Carrillo C, Line J, Rojas O (2014) Microemulsion systems for fiber deconstruction into cellulose nanofibrils. Appl Mater Interfaces 6:22622

Fukuzumi H, Saito T, Kumamoto Y, Iwata T, Isogai A (2009) Transparent and high gas barrier films of cellulose nanofibers prepared by TEMPO-mediated oxidation. Biomacromolecules 10:162

Fukuzumi H, Saito T, Okita Y, Isogai A (2010) Thermal stabilization of TEMPO-oxidized cellulose. Polym Degrad Stab 9:1502

Habibi Y (2014) Key advances in the chemical modification of nanocelluloses. Chem Soc Rev 43:1519

Henriksson M, Henriksson G, Berglund LA, Lindström T (2007) An environmentally friendly method for enzyme-assisted preparation of microfibrillated cellulose (MFC) nanofibers. Eur Polym J 43:3434

Herrera M, Sirvio J, Mathew A, Oksman K (2016) Environmental friendly and sustainable gas barrier on porous materials: nanocellulose coatings prepared using spin-and dip-coating. Mater Des 93:19

Herzele S, Veigel S, Liebner F, Zimmermann T, Gindl-Altmutter W (2016) Reinforcement of polycaprolactone with microfibrillated lignocellulose. Ind Crops Prod 93:302

Isogai A, Saito T, Fukuzumi H (2011) TEMPO-oxidized cellulose nanofibers. Nanoscale 3:71

Jonoobi M, Oladi R, Davoudpour Y, Oksman K, Dufresne A, Hanzeh Y, Davoodi R (2015) Different preparation methods and properties of nanostructured cellulose from various natural resources and residues: a review. Cellulose 22:935

Lange J, Wyser Y (2003) Recent innovations in barrier technologies for plastic packaging - a review. Packag Technol Sci 16:149

Ma P, Zhai H, Law K-N, Daneault C (2010) Influence of oxidation and cationization on the properties of thermomechanical pulp fibers. Tappi J 9:36

Ma P, Fu S, Zhai H, Law K, Daneault C (2012) Influence of TEMPO-mediated oxidation on the lignin of thermomechanical pulp. Bioresour Technol 118:607
Nair S, Yan N (2015) Effect of high residual lignin on the thermal stability of nanofibrils and its enhanced mechanical performance in aqueous environments. Cellulose 22:3137

Nechyporchuk O, Belgacem M, Bras J (2016) Production of cellulose nanofibrils: a review of recent advances. Ind Crops Prod 93:2

Okita Y, Saito T, Isogai A (2009) TEMPO-mediated oxidation of softwood thermomechanical pulp. Holzforschung 63:529

Okita Y, Saito T, Isogai A (2010) Entire surface oxidation of various cellulose microfibrils by TEMPO-mediated oxidation. Biomacromolecules 11:1696

Pandey J, Nakaito A, Takagi H (2013) Fabrication and applications of cellulose nanoparticle-based polymer composites. Polym Eng Sci 53:1

Rinaldi R, Jastrzebski R, Clough M, Ralph J, Kennema M, Bruijinincx P, Weckhuysen B (2016) Paving the way for lignin valorisation: recent advances in bioengineering, biorefining and catalysis. Angew Chem Int Ed 55:8164

Rojo E, Peresin M, Sampson W, Hoeger I, Vartiainen J, Laine J, Rojas O (2015) Comprehensive elucidation of the effect of residual lignin on the physical, barrier, mechanical and surface properties of nanocellulose films. Green Chem $17: 1853$

Saito T, Hirota M, Tamura N, Isogai A (2010) Oxidation of bleached wood pulp by TEMPO/ $\mathrm{NaClO} / \mathrm{NaClO}_{2}$ system: effect of the oxidation conditions on carboxylate content and degree of polymerization. J Wood Sci 56:227

Sehaqui H, Liu A, Zhou Q, Berglund L (2010) Fast preparation procedure for large, flat cellulose and cellulose/inorganic nanopaper structures. Biomacromolecules 11:2195

Szczesniak L, Rachocki A, Tritt-Goc J (2008) Glass transition temperature and thermal decomposition of cellulose powder. Cellulose 15:445

Uhlig M, Fall A, Wellert S, Lehmann M, Prévost S, Wågberg L, von Klitzing R, Nyström G (2016) Two-dimensional aggregation and semidilute ordering in cellulose nanocrystals. Langmuir 32:442

Vänskä E, Vihelä T, Peresin MS, Vartiainen J, Hummel M, Vuorinen T (2016) Residual lignin inhibits thermal degradation of cellulosic fiber sheets. Cellulose 23:199

Watanabe E, Tamura N, Fujisawa S, Saito T, Habu N, Isogai A (2013) Stability of $(1 \rightarrow 3)$ - $\beta$-polyglucuronic acid under various $\mathrm{pH}$ and temperature conditions. Carbohydr Polym 97:413

Yang H, Yan R, Chen H, Lee D, Zheng C (2007) Characteristics of hemicellulose, cellulose and lignin pyrolysis. Fuel First $86: 1781$ 